\title{
Findings from the Families on Track intervention pilot trial for children with fetal alcohol spectrum disorders and their families
}

\author{
Christie L. M. Petrenko, Ph.D. ${ }^{1}$, Mary E. Pandolfino, B.A. ${ }^{1}$, and Luther K. Robinson, M.D. ${ }^{2}$ \\ ${ }^{1}$ Mt. Hope Family Center, University of Rochester \\ ${ }^{2}$ Women and Children's Hospital of Buffalo, University at Buffalo
}

\begin{abstract}
Background-Individuals with fetal alcohol spectrum disorders (FASD) are at high risk for costly, debilitating mental health problems and secondary conditions, such as school disruption, trouble with the law, and substance use. The study objective was to pilot a multi-component intervention designed to prevent secondary conditions in children with FASD and improve family adaptation.
\end{abstract}

\begin{abstract}
Methods-Thirty children with FASD or prenatal alcohol exposure (PAE) (ages 4 to 8) and their primary caregivers were enrolled. Families were randomized to either the Families on Track Integrated Preventive Intervention or an active control of neuropsychological assessment and personalized community referrals. The 30 -week intervention integrates scientifically validated bimonthly, in-home parent behavioral consultation and weekly child skills groups. Outcomes measured at baseline and follow-up post-intervention included intervention satisfaction, child emotional and behavioral functioning, child self-esteem, caregiver knowledge of FASD and advocacy, caregiver attitudes, use of targeted parenting practices, perceived family needs met, social support, and self-care. Data analysis emphasized calculation of effect sizes and was supplemented with analysis of variance techniques.
\end{abstract}

Results-Analyses indicated that families participating in the intervention reported high program satisfaction. Relative to comparison group outcomes, the intervention was associated with medium to large effects for child emotion regulation, self-esteem, and anxiety. Medium-sized improvements in disruptive behavior were observed for both groups. Medium and large effects were seen for important caregiver outcomes: knowledge of FASD and advocacy, attributions of behavior, use of antecedent strategies, parenting efficacy, family needs met, social support, and self-care.

Conclusions-This pilot study yielded promising findings from the multicomponent Families on Track Integrated Preventive Intervention for child and caregiver outcomes. An important next step is to complete a randomized control trial of the Families on Track program with a larger sample fully representative of this underserved clinical population with built-in study of implementation parameters.

Corresponding author: Christie L. M. Petrenko, Ph.D., Mt. Hope Family Center, University of Rochester, 187 Edinburgh St., Rochester, NY 14608, USA. Phone: 585-275-2991 x 241. Fax: 585-454-2972. Christie.petrenko@ rochester.edu. 


\section{Keywords}

fetal alcohol spectrum disorders; prenatal alcohol exposure; intervention; secondary conditions

\section{Introduction}

Fetal alcohol spectrum disorders (FASD) represent a range of conditions associated with prenatal alcohol exposure (PAE). The prevalence of FASD is estimated at $1 \%$ conservatively (Sampson et al., 1997) and up to $4.8 \%$ using active case ascertainment (May et al., 2014). Although the pattern varies by individual, common areas of impairment include intellectual functioning, learning and memory, visuospatial skills, executive functioning, social communication, and emotional and behavioral regulation (Mattson et al., 2011). Individuals with FASD are at high risk for mental health problems and secondary conditions, such as school disruption, trouble with the law, confinement, substance use problems, and difficulties with independent living and employment (Streissguth et al., 2004). Although rates of mental health problems are high across the lifespan, the onset of most secondary conditions occurs during adolescence and adulthood, suggesting the promise of preventing or mitigating these by mobilizing tailored, appropriate intervention in the preschool and elementary school years.

FASD places a heavy burden on affected individuals, families, and society (Popova et al., 2016). Caregivers experience serious emotional and practical stressors arising from efforts to manage their children's disabilities, obtain needed services, and cope with pervasive limitations in knowledge of FASD among providers and community members (Olson et al., 2009; Petrenko et al., 2014a,b; Petrenko et al., manuscript under review; Ryan et al., 2006).

Developing and testing appropriate interventions are imperative tasks. The current study objective was to pilot a multi-component intervention for preschool and early school-aged children with FASD and their families designed to prevent secondary conditions and improve family adaptation.

\section{Evidence Base for FASD Interventions}

Although still limited, the evidence base for interventions for individuals with FASD and their families has grown over the last decade (Reid et al., 2015). Most studies have focused on interventions for preschool and school-aged children (ages 3-12). Domains targeted have included attention, self-regulation, social and adaptive skills, academic achievement, parent training, and externalizing behavior (Petrenko, 2015). Results demonstrate that children with FASD can learn new skills and benefit from interventions that are appropriately tailored to their neurodevelopmental strengths and weaknesses. Many child-focused interventions have included training for caregivers or teachers to aid in skill coaching and generalization.

Although significant improvements have been documented, children in intervention groups often continue to function below expected levels for their age. It seems clear that environmental modifications and continued skill development across the lifespan may be required for many individuals with FASD. 
Much work remains to establish and increase access to evidence-based interventions for individuals with FASD and their families (Olson, 2016). An especially important task is identifying effective approaches to prevent the onset or mitigate the severity of mental health problems and secondary conditions. Existing theory and research provides important guidance for developing preventive interventions for this population.

\section{Prevention of Secondary Conditions: Theoretical and Empirical Considerations}

Developmental psychopathology provides a useful theoretical basis for conceptualizing preventive interventions (Cicchetti \& Toth, 2009; Toth et al., 2016) and unifies existing theories for FASD interventions (Kodituwakku, 2009; Olson et al., 2009; Petrenko, 2015). From this perspective, preventive interventions target relevant reciprocal transactions among risk and protective factors across ecological levels (e.g., individual, family, community, culture) and multiple levels of analysis (e.g., genetic, neural, physiological, behavioral, interpersonal) to promote more adaptive developmental trajectories. Exerting appropriate, targeted influences to alter a child's developmental trajectory onto more adaptive pathways enhances the likelihood the child will experience subsequent successful adaptation. As children move from preschool to the early school years, the most salient developmental tasks involve achieving flexible self-regulation and competence in major life spheres such as school and social functioning (Sroufe, 2013). Children with FASD often struggle in these areas (Kable et al., 2015). This means preventive interventions focused on this crucial developmental period are needed to promote current and future adaptation.

Research and theory suggest important risk and protective factors during these early years relating to development of secondary conditions (Olson \& Montague, 2011). These could be targeted by preventive interventions. In one large, cross-sectional clinical sample, the most salient malleable protective factors related to reduced odds of secondary conditions included an early FASD diagnosis (before age 6), a stable and nurturing home during middle childhood, receipt of appropriate developmental services, and not being the victim of violence (Streissguth et al., 2004). Olson and colleagues (2009) identified additional relevant family-level risk and protective factors including parent-child interaction patterns, caregiver cognitive appraisal, parenting self-efficacy, parent stress, and family resource needs.

Community service systems also pose significant risk and protective factors for individuals with FASD, such as their level of awareness about FASD, and the availability, accessibility, and implementation of services. Recent qualitative research documents that systems-level barriers contribute to secondary conditions in individuals with FASD and interact with the family system, increasing emotional and practical stressors for caregivers (Baskin et al., 2016; Petrenko et al., 2014b; Petrenko et al., manuscript under review).

Qualitative research with key stakeholders has suggested ideal characteristics of preventive interventions for individuals with FASD and their families (Baskin et al., 2016; Petrenko et al., 2014a). Interventions need to consider the "whole person" and provide comprehensive, individualized, and well-coordinated supports to the person with FASD, their family, and across systems to promote successful adaptation. Ideally, services and supports should take a preventive or proactive stance (vs. waiting for a person to fail or symptoms to appear) and be available across the lifespan. These ideal intervention characteristics correspond well with 
principles of the positive behavior support (PBS) framework (Carr et al., 2002; Koegel et al., 1996; Olson \& Montague, 2011; Petrenko, 2015), and recent movement toward use of integrated care models (SAMHSA-HRSA Center for Integrated Health Solutions, 2013).

\section{The Families on Track Integrated Preventive Intervention Program}

The Families on Track Integrated Preventive Intervention Program targets key risk and protective factors for children with FASD or PAE (ages 4 to 8) and their families to prevent secondary conditions and improve family adaptation. The program begins with a neuropsychological and diagnostic evaluation to promote the protective factor of early diagnosis and to identify the child's neuropsychological profile. Children and their families then receive a 30-week multi-component intervention that integrates two existing empirically-validated programs: the preschool/kindergarten Promoting Alternative THinking Strategies (PATHS) curriculum (Domitrovich et al., 2005) and the Families Moving Forward (FMF) Program for caregivers of children with FASD created by Olson and colleagues (Bertrand, 2009, Study \#5).

The PATHS curriculum was developed for children in grades preK-6 to prevent violence, aggression, and other behavioral problems by promoting social competence (Domitrovich et al., 2005; Kusche \& Greenberg, 1994). Found effective with students in regular and special education classrooms, it has been recognized nationally by multiple organizations as a model program (Greenberg et al., 1998). For the Families on Track Program, the pre-K/ Kindergarten curriculum was selected to best match the developmental level and attentional abilities of children (ages 4-8) with FASD. The curriculum was delivered over 30 weeks in weekly skills groups that included children with FASD and typically developing positive peer models. Skills taught in the PreK-K PATHS curriculum emphasize self-control, emotional understanding, positive self-esteem, peer relationships, and interpersonal problem solving skills. These skills are particularly relevant for age-appropriate, crucial developmental tasks, and successful attainment of these skills in childhood are related to better outcomes in adulthood (e.g., Jones, Greenberg, \& Crowley, 2015; Moffitt et al., 2011). These skills are also particularly critical to target for children with FASD as they often represent areas of impairment relative to same-aged peers (e.g., Kable et al., 2015; KullyMartens et al., 2012). Teacher implementation and generalization of skills have been found to be important predictors of improved outcomes in prior PATHS studies in the school environment (Domitrovich et al., 2010; Kam et al., 2003); the Families on Track program teaches caregivers how to generalize PATHS skills at home to promote this finding.

The innovative FMF Program targets relevant individual and transactional family-level risk and protective factors including parent-child interaction patterns, caregiver cognitive appraisal, parenting self-efficacy, caregiving stress, family resource needs, and a factor central to family adaptation: child behavior problems. Targeting these factors promotes a stable and nurturing home and reduces the risk of violence toward the child, which have been identified as universal protective factors against secondary conditions in FASD (Streissguth et al., 2004). Grounded in developmental and family systems theories, the caregiver-focused FMF Program is designed to modify specific parenting attitudes and responses to children's problem behaviors via integration of a PBS approach taught to 
families, cognitive behavioral strategies, and motivational interviewing (Bertrand, 2009;

Olson et al., 2009; Olson \& Montague, 2011). By helping caregivers interpret their children's behavior from a neurodevelopmental perspective (called "reframing" in FMF), it is theorized they will develop a more positive and realistic appraisal of the child, use more effective antecedent-based behavioral strategies to promote adaptive child functioning and decrease challenging behavior, and feel more efficacious as a parent. Recent cross-sectional research supports associations among these constructs (Petrenko et al., 2016). The FMF Program was found to be acceptable to caregivers and clinicians, and significantly met important, unmet family needs in previous trials (Bertrand, 2009). Intervention families also demonstrated increased knowledge of FASD and advocacy skills, specific caregiving attitudes (including positive appraisal of the child and parenting efficacy), increased selfcare, use of targeted parenting practices, and decreased child disruptive behavior (Bertrand, 2009; Olson \& Sparrow, unpublished presentation 2016). The FMF Program also includes targeted consultation with the child's school and other community providers, and efforts to link families with appropriate community services and supports. These components of the FMF Program target transactional risk and protective factors between families and service systems relating to FASD knowledge and advocacy, and the accessibility and implementation of services.

The primary study objective was to pilot the Families on Track Program, assessing the magnitude of intervention effects to aid in planning larger-scale intervention/implementation trials. Outcomes assessed included intervention uptake and satisfaction, child emotional and behavioral functioning, child self-esteem, caregiver knowledge of FASD and advocacy, caregiver attitudes, use of targeted parenting practices, perceived family needs met, social support, and self-care. It was hypothesized that families completing the Families on Track Program would have better outcomes relative to an active control comparison condition.

\section{Materials and Methods}

\section{Study Design}

This study was a small-scale pilot randomized controlled trial with two groups: 1) Families on Track, or 2) Active control, which involved a neuropsychological evaluation and customized community referrals. Participants were recruited each summer for two years $(2013,2014)$. Randomization was stratified by child sex and age (4-5, 6-8). Data are from baseline and immediate post-intervention ( $\sim 1$ year after baseline). The University Institutional Review Board approved all procedures.

\section{Participants}

Participants included 30 children with an FASD or PAE and their primary caregivers. Families were recruited through provider referrals, brochures and flyers, and presentations at local parent support groups. Families who met eligibility criteria reported learning about the study from the following sources: FASD Diagnostic Clinic (run by third author; $n=14$ ); specialty or general medical practice $(n=7)$; mental health provider $(n=4)$; parent support group $(n=3)$; and colleague/friend $(n=2)$. Oral consent was obtained prior to eligibility screening. 
To be eligible, children: 1) attained an FASD diagnosis based on Revised Institute of Medicine guidelines (Hoyme et al., 2005) or had confirmed history of PAE; 2) were between 4 and 8 years old; 3 ) lived within a reasonable distance of two New York study sites ; and 4) were expected to remain in their current placement for the study duration ( $\sim 18$ months, including 9-month intervention and follow-up time points). All children were evaluated by a dysmorphologist with expertise in FASD (third author), and received a neuropsychological evaluation. Just over 40 percent of children met criteria for a diagnosis of fetal alcohol syndrome (FAS) or partial FAS. Remaining participants had confirmed PAE and some evidence of CNS dysfunction, but lacked characteristic facial features. Given the overarching intervention goal to prevent secondary conditions and the sample's young age, children with PAE were not required to meet full criteria for alcohol-related neurodevelopmental disorder (ARND; requiring at least 3 domains of significant CNS impairment) to participate. All of these children would meet the revised 2016 Hoyme criteria for ARND (Hoyme et al., 2016). Children were excluded from the study if they had moderate to severe intellectual disabilities (IQ<55), lacked sufficient English proficiency, or had severe physical or mental conditions precluding them from safe participation.

The Families on Track program was considered a "value-added" intervention and families in either group were not precluded from participating in other intervention programs. At the baseline assessment, half of families $(n=15)$ reported receiving outpatient child, caregiver, or family interventions within the last 12 months to address child mental health or behavioral problems. An additional 8 families $(26.7 \%)$ reported their child received counseling services within the school setting only.

Following initial eligibility determinations, informed consent, permission, and assent (for children 27 ) were obtained. Families identified a target child for research purposes if they had more than one eligible child.

\section{Interventions}

Assessment and referral-All children underwent a neuropsychological assessment (see Table 1 for measures) upon enrollment. An integrated report for each child was prepared, including individualized recommendations. The first author gave personalized feedback to each caregiver in a single 60-min session. Neuropsychological assessment with an FASD-knowledgeable provider is very limited in the study region; this level of service should be considered an active comparison.

Families on Track Integrated Preventive Intervention Program-The 30-week Families on Track Program consists of in-home parent consultation using the FMF Program (Bertrand, 2009; Olson \& Montague, 2011) and weekly child skills groups using the PATHS curriculum (Domitrovich et al., 2005) (see Table 2 for topics). All intervention components were delivered by the first author and advanced graduate students in clinical or school psychology. All interventionists underwent 50+ hours of training in motivational interviewing, the FMF Program, and the PATHS curriculum. Fidelity was monitored in weekly individual and group supervision meetings (2-3 hrs/week) by the first author. 
Two child skills groups were run each year, with 6 to 10 children per group. Groups included 3-4 target children with FASD, siblings in the target age range (with or without FASD), and typically developing positive peers. Groups were co-led, with 1 to 2 undergraduates assisting. Groups were held weekly on Saturdays at University or community locations. The implementation of the PATHS curriculum was modified for the small-group setting (PATHS is traditionally delivered in an educational setting). Lessons from the preK/K PATHS curriculum were delivered as specified in the intervention manual. The structure of the skills group was formatted to provide opportunities for prosocial interaction and skill practice. The typical structure for each 90-minute session was as follows: 1) welcome activity, 2) PATHS lesson, 3) snack, 4) extension activities (usually 2-3) to create opportunities to practice the skill taught, and 5) free play. Group-wide PBS was utilized and individualized behavior plans were created for children who needed additional support to participate in the group setting.

FMF sessions were delivered every other week in families' homes for 90 minutes. The FMF Specialist working with the family was also typically the child's skills group leader. The FMF Specialist reviewed with caregivers the skills children were learning in group and discussed ways to best generalize these skills within the home environment. Explicit instruction in the Turtle Technique, which is used extensively beginning in week 11 with the children in the PATHS curriculum to teach self-control, was integrated into the session outline for the "Understanding the Whole Child" session in the FMF curriculum for this project (see Table 2). Specialists also considered careful observations from child skills groups when assisting parents with developing behavior plans for the child. Even in the few cases in which the FMF Specialist was not the child's group leader, all FMF Specialists were involved in weekly group supervision and were well informed about the skills being taught and the progress of the child from the family on their caseload.

\section{Research Visit Procedures}

Children and their caregivers completed two research visits, one at baseline and one approximately 1 year later. Trained research assistants completed visits, which averaged 2 hours. Attempts were made to keep research assistants blind to intervention condition.

\section{Measures}

Measures of child functioning-Berkeley Puppet Interview (BPI; Measelle et al., 1998): a semi-structured interview of young children's (ages 4 to 8 ) perceptions of themselves and their environments. The interview is conducted through two puppets, and the child indicates which of two opposing statements is most like him/her. Responses are coded on a 7-point scale with scores 1-3 reflecting degree of endorsement of the negative option, 4 reflecting endorsement of both options, and 5-7 reflecting degree of endorsement of the positive option. Internal consistency was adequate for the selected scales: Prosocial (baseline $a=0.76$, follow-up $a=0.70$ ), Overanxious (baseline $a=0.80$, follow-up $a=0.79$ ), and Conduct Problems ${ }^{1}$ (baseline $a=0.82$, follow-up $a=0.70$ ). Inter-rater reliability was

\footnotetext{
1Item \#35 "mean to animals" was omitted from the scale due to poor internal consistency with other items for this sample.
} 
adequate: mean intra-class correlation coefficients (ICC) of 0.95 (range 0.86-1.00) at baseline and 0.96 (range 0.78-1.00) at follow-up.

Eyberg Child Behavior Inventory (ECBI; Eyberg \& Pincus, 1999): a 36-item parent rating scale measuring disruptive behavior in children ages 2-16. This study used the Intensity scale; items are rated on a 7-point scale to indicate behavior frequency. Higher scores (Tscores; $\mathrm{M}=50, \mathrm{SD}=10)$ indicate more behavior problems. Internal consistency is high in normative $(a=.95)$ and current (baseline $a=0.94$, follow-up $a=0.93$ ) samples.

Emotion Regulation Checklist (ERC; Shields \& Cicchetti, 1997): a 24-item parent report checklist assessing children's ability to regulate emotions and behavior. Items are rated on a 4-point scale from $1=$ never to $4=$ always. The Lability/Negativity subscale assesses a lack of flexibility, mood lability, and dysregulated negative affect (high scores reflect dysregulated, negative affect). The Emotion Regulation subscale measures situationally appropriate affective displays, empathy, and emotional self-awareness (high scores reflect better emotion regulation). Internal consistency was adequate in the current sample (Lability/Negativity baseline $a=0.90$, follow-up $a=0.91$; Emotion Regulation baseline $a=0.79$, follow-up $a=0.72$ ).

Impairment Rating Scale (IRS; Fabiano et al., 2006): a practical parent report of child impairment. Parents consider the child's functioning in seven domains and place an $\mathrm{X}$ on a line that signifies the child's level of impairment from 1 (no problem/definitely does not need treatment or services) to 7 (extreme problem/definitely needs treatment or special services). The current study examined Self-Esteem and Overall Impairment.

\section{Measures of caregiver functioning-Knowledge and Advocacy Scale (K\&A;}

Bertrand, 2009; Olson et al., 2009): assesses parent knowledge about issues related to alcohol-related disabilities and advocacy for children with special needs. The K\&A has 42 items; response options to items vary and include True/False, level of agreement, and multiple choice. The $\mathrm{K} \& \mathrm{~A}$ is scored by calculating the total number of items correct. For level of agreement items, responses are scored correct if receiving the correct valence (e.g., credit for "agree" or "strongly agree" if item statement is true). Internal consistency is adequate in this sample (baseline $a=0.84$, follow-up $a=0.85$ ).

Parenting Practices Interview (PPI; Petrenko et al., 2016): a qualitative interview designed to assess parenting strategies and parental attributions for child misbehavior. PPI interviews were audio recorded and transcribed verbatim. Interviews were independently coded by at least two trained coders and final codes were determined by consensus. Parental attributions of behavior were coded on an 8-point scale reflecting the degree of willful versus neurodevelopmental attributions for behavior $(1=$ strongest neurodevelopmental, $8=$ strongest willful). The use of antecedent strategies was coded on a 5-point scale (1=systematic use, $5=$ no mention). Inter-rater reliability among independent raters prior to consensus were: Attribution (baseline ICC $=0.92$, follow-up ICC $=0.86$ ) and Antecedents (baseline $\mathrm{ICC}=0.85$, follow-up ICC $=0.58$ ). Including the final consensus rating, ICC estimates were: Attribution (baseline ICC $=0.95$, follow-up ICC $=0.94$ ) and Antecedents (baseline ICC $=0.91$, follow-up $\mathrm{ICC}=0.83$ ). 
Family Needs Met (Olson et al., 2009): based on a measure developed for traumatic brain injury (Kreutzer et al., 1988), the measure includes items relating to common needs parents of children with alcohol-related disabilities sometimes have and assesses the degree to which caregivers perceive these needs have been met. Each need is rated on a 4-point scale ( $1=$ not at all met, $4=\mathrm{a}$ great deal met). The total score reflects the average response across items. Internal consistency in the current sample was high (baseline $a=0.95$, follow-up $a=0.94$ ).

Parenting Sense of Competence (PSOC; Johnston \& Mash, 1989): a 16-item caregiver selfreport measure of parenting efficacy and satisfaction. Items are rated on a 6-point scale ("strongly disagree" to "strongly agree"). The scale assesses two factors: Satisfaction, or extent to which the individual enjoys the parenting role (low scores=low satisfaction), and Efficacy, or perceived competence in the parenting role (low scores=high efficacy). Internal consistency is adequate in normative (Efficacy $a=0.76$; Satisfaction $\mathrm{a}=0.75$ ) and current (Efficacy baseline $a=0.72$, follow-up $a=0.79$; Satisfaction baseline $a=0.80$, follow-up $a=0.76$ ) samples.

Parenting Stress Index, Fourth Edition, Short Form (PSI-4-SF; Abidin, 2012): a 36-item, abbreviated inventory, evaluating the magnitude of stress in the parent-child system. The Parental Distress scale was used. Scores are T-scores $(\mathrm{M}=50, \mathrm{SD}=10)$, with higher scores reflecting greater stress. Internal consistency is adequate in normative $(a=.95)$ and current (baseline $\mathrm{a}=0.88$, follow-up $\mathrm{a}=0.92$ ) samples.

Perceived Social Support Scale (PSSS; Carr \& O'Reilly, 2000): a 20-item self-report questionnaire that assesses perceived support from family, friends, significant others and involved professionals. Items were rated on a 7-point scale and responses were averaged to yield an overall score, with higher scores indicating greater perceived support. Internal consistency was high in the current sample (baseline $a=0.92$, follow-up $a=0.93$ ).

Self-care. At post-intervention, caregivers were asked to compare their current level of selfcare to their level of self-care at baseline and select a response on a 5 -point scale $(1=\mathrm{a}$ lot less self-care, $3=$ no change in self-care, to $5=$ a lot more self-care).

Child and caregiver demographic information-Baseline Caregiver Interview (BCI): contains questions about child and family demographics, the child's health, education, and placement history.

Intervention satisfaction-Client Satisfaction Questionnaire (CSQ; Larsen et al., 1979): a widely used 8-item questionnaire to assess client satisfaction with services received. Items are rated on a 4-point scale. Items were summed, with higher scores reflecting greater satisfaction. Internal consistency was adequate in this sample $(a=0.90)$.

Parent Evaluation Inventory-FOT (PEI-FOT): based on an adaptation of the original PEI (Kazdin et al., 1987), which has been used to assess parent self-report of treatment progress and earlier FMF Program studies. This version of the PEI has 17 items. Items are rated on 5point scale $(0=$ most negative outcome, $4=$ most positive outcome). 


\section{Data Analysis}

All data were entered into SPSS 23 and manually re-checked to confirm accuracy. Variables were examined for presence of outliers and assumptions for analysis of variance techniques (i.e., normality, homogeneity of variance) were assessed within groups. An outlier was considered for exclusion from analysis if it fell outside the range of values for both groups and there was a meaningful interpretation for its unusualness. Transformations were considered for variables with marked deviations in normality or homogeneity of variance.

Analyses emphasized the calculation of effect sizes within and between groups. Two measures of effect size are reported. Cohen's d (Cohen, 1988) was used to measure within group change from pre-intervention to post-intervention. For consistency, the direction of each effect size was coded as positive if it reflected improved functioning and negative if it reflected worsening over time. A second index, the pretest-posttest-control (PPC) effect size or $\mathrm{d}_{\mathrm{ppc}}$, was used to quantify change in repeated measurement between intervention and comparison groups (Morris, 2008). The direction of effect is reported as positive if it reflects greater relative improvement for the intervention group and negative if it reflects greater improvement for the comparison group. For both effect size indices, 0.2 represents a small effect, 0.5 a medium effect, and 0.8 a large effect.

Estimates of effect size were supplemented with significance testing using analysis of variance techniques with one within-subjects factor (pre-intervention and post-intervention outcome measurement) and one between-subjects factor (intervention group status). Due to small sample size, these analyses are powered to detect only large effect sizes.

\section{Results}

\section{Participant Characteristics and Flow Diagram}

The study flow diagram is presented in Figure 1. Three families declined the intervention for logistical reasons ( 2 decided they lived too far to drive on a weekly basis, 1 scheduling conflict and transportation difficulty); all three completed at least a portion of the postintervention research visit (100\% caregiver, 33\% child). For analysis, data from the 3 families who declined the intervention were combined with the 9 families in the comparison group with post-intervention data, resulting in 15 families who received the Families on Track intervention and 12 who did not. The modest sample size precluded meaningful intent-to-treat analyses. Baseline participant characteristics can be found in Table 3 by group. There were no statistically significant group differences in participant characteristics or baseline levels of functioning.

\section{Intervention Uptake and Satisfaction}

Of 16 families starting intervention, 15 families completed the program (93.8\%). Of families remaining in intervention, 15 caregivers completed all 12 core sessions (100\%) of the FMF Program. As specified for the FMF Program, families were offered individualized sessions and a school consultation visit. All 15 caregivers completed one individualized session (100\%), 12 completed 2 individualized sessions (80\%), and 11 completed the school consultation (73\%). Reasons for declining school consultation included: homeschooling (1), 
parent deemed it unnecessary (2), and school scheduling difficulties (1). Children attended an average of 22.73 of 30 skills group sessions (range 13-28).

On the CSQ, caregivers reported a mean satisfaction score of $30.67(\mathrm{SD}=2.29)$ out of 32 (range 23-32). The only caregiver with a satisfaction score below 30 had an unusual intervention course. On the PEI-FOT, caregivers reported a high level of enjoyment of the program (mean $=3.73, \mathrm{SD}=0.59$ ) and felt that they could apply what they learned from sessions (mean $=3.60, \mathrm{SD}=0.63$ ). They rated their relationship with their FMF Specialist highly (mean $=3.93, \mathrm{SD}=0.26$ ) and felt the Specialist understood their feelings and problems with their child (mean=3.87, $\mathrm{SD}=0.52$ ). Caregivers reported that children generally looked forward to coming to group (mean=3.64, $\mathrm{SD}=0.63$ ) and learned new skills (mean=3.36, $\mathrm{SD}=0.84$ ). However, they perceived that children had relatively more difficulty applying what they learned (mean $=2.79, \mathrm{SD}=0.80$ ) and thus were moderately improved (mean=2.87, $\mathrm{SD}=1.06)$.

\section{Assessment of Pre-Post Outcomes Associated with the Families on Track Program}

Child outcomes-Means, standard deviations, within-group effect sizes, and between group effect sizes for child-related outcomes are in Table 4. Results from significance testing are in Table 5. For child-related outcomes, the largest effect associated with the Families on Track Program was found for caregiver-reported change in child emotion regulation (ERC $\mathrm{d}_{\mathrm{ppc}}=1.18$ ), a significant group difference. This effect reflected a medium to large improvement in emotion regulation for the intervention group and medium-sized decrement for the comparison group.

A medium to large group effect size for caregiver-reported self-esteem was found (IRS self esteem $d_{p p c}=0.77$ ), which was not statistically different between groups. The self-esteem variable was log-transformed due to marked deviations from normality. Over time, the intervention group showed little mean change in self-esteem ratings, remaining just under the clinical cut-off. In contrast, on average the comparison group started off with better ratings, but showed a large decline in self-esteem reaching the clinical cutoff by postintervention. Significance testing identified a significant effect of time; all children, on average, showed some declines in self-esteem over time.

A medium to large group effect was found for child-reported anxiety symptoms (BPI Overanxious $\mathrm{d}_{\mathrm{ppc}}=0.75$ ), which did not reach statistical significance. Children receiving the intervention reported a somewhat higher level of anxiety symptoms at pre-intervention with improvement over time $\left(\mathrm{d}_{\text {within }}=0.80\right)$. In contrast, comparison children showed minimal change in self-reported anxiety.

Minimal to small group effects were identified for caregiver report of child disruptive behavior (ECBI Intensity), negative affect/lability (ERC), and global impairment (IRS), and in child report (BPI) of prosocial skills and conduct problems. None of these between-group effects (time $\times$ group interaction) were statistically significant. For the disruptive behavior (ECBI Intensity) and Negative Affect/Lability (ERC) variables, examination of within-group effect sizes reveals that both groups demonstrated similar levels of improvement (small to 
medium effects). Main effects of time were statistically significant for both of these variables.

Caregiver outcomes-Means, standard deviations, within-group effect sizes, and between group effect sizes for caregiver outcomes are in Table 6. Significance testing results are in Table 7. The largest caregiver effect associated with the Families on Track Program was for knowledge of FASD and advocacy $\left(K \& A d_{p p c}=1.02\right)$, a statistically significant effect. Caregivers receiving the intervention demonstrated a large increase in knowledge over time, while comparison caregivers showed little to no change.

Group effect size for Family Needs Met approached a large effect $\left(\mathrm{d}_{\mathrm{ppc}}=0.72\right)$, with a significant group difference ${ }^{2}$. Those receiving the intervention reported a very large increase in perceived needs met, while comparison families showed a small to medium increase in perceived needs met.

Medium group effects were identified for targeted treatment processes of parental attributions of behavior ("reframing") (PPI Attributions $\mathrm{d}_{\mathrm{ppc}}=0.61$ ) and use of antecedent strategies (PPI Antecedents $d_{p p c}=0.54$ ). Neither of these effects reached statistical significance. The intervention group transitioned from more willful attributions of child behavior to an increase in neurodevelopmental attributions, a change in the expected direction on a theorized mechanism of change. In contrast, comparison caregivers showed no change in parental attributions. For antecedent strategy use, the intervention group exhibited a small increase in antecedent use, while the comparison group displayed a small decline in use.

Medium-level group effects in the expected direction were documented for the important constructs of parenting self-efficacy (PSOC $d_{p p c}=0.46$ ) and social support (PSSS $d_{p p c}=0.57$ ). Neither of these effects reached statistical significance. For both measures, the intervention group displayed small increases while the comparison group showed small declines. Compared to normative samples, group means were generally within the average range.

Changes in self-care relative to baseline were assessed at post-intervention. A small to medium group difference $(\mathrm{d}=0.48)$ was found, with the intervention group (mean $=3.73$, $\mathrm{SD}=0.88$ ) reporting a somewhat greater increase in self-care relative to the comparison group (mean $=3.33, \mathrm{SD}=0.78$ ), though this effect was not statistically significant $(\mathrm{t}(25)=-1.23, p=0.23)$.

Small group effects were identified for parenting satisfaction (PSOC satisfaction $\mathrm{d}_{\mathrm{ppc}}=0.19$ ) and parent distress (PSI PD d $\mathrm{ppc}=0.22$ ). These effects were not statistically significant. Both groups attained scores within the average range and showed minimal to small change over time.

\footnotetext{
${ }^{2}$ Data from one caregiver in the intervention group was omitted from analysis of the Family Needs Met measure due to an extremely low score at post-intervention. The child left this pre-adoptive placement around the time of post-intervention assessments. The family's scores were not outliers on any other outcome measure.
} 


\section{Discussion}

The overall aims of this study were to pilot the Families on Track Integrated Preventive Intervention with young children with FASD or PAE and their families and assess the magnitude of intervention effects to guide future larger-scale trials. The Families on Track Program is one of the most intensive interventions studied to date for children with FASD or PAE. Findings suggest that this coordinated, multicomponent intervention yields a broader range of effects for important child and caregiver outcomes than neuropsychological assessment and targeted referral to community services. Specifically, similar improvements in child disruptive behavior were seen in the intervention and active control groups, which were concordant with the intervention group (but not standard of care group) in a prior FMF trial (Bertrand, 2009). Although child behavior effects were similar, the high intensity Families on Track Program produced greater improvements even relative to the active control, in the crucial outcomes of caregiver knowledge, caregiver attitudes, targeted parenting practices, parenting efficacy, support, self-care, and child regulation and selfesteem. Effect sizes were medium in magnitude or greater on these outcomes and support investment in future larger-scale trials of the program.

This small-scale trial also provided a preliminary empirical test of proposed theoretical mechanisms underlying the FMF Program (Bertrand, 2009; Olson et al., 2009). Prior crosssectional analyses from baseline data with this sample found support for theorized associations between parental attributions, antecedent strategies, and parenting efficacy (Petrenko et al., 2016). Results from this trial showed that a tailored intervention targeting these outcomes can produce meaningful change in expected ways. As predicted, the FMF Program resulted in large increases in caregiver knowledge, and medium changes in neurodevelopmental attributions, use of antecedent strategies, and parenting efficacy. Furthermore, the relative magnitude of effect sizes across caregiver outcomes suggests that the psychoeducation and cognitive behavioral strategies used within the FMF Program may have the largest family impact. The development of the PPI was instrumental in documenting change on these important, treatment-relevant constructs and initiating needed research on mechanisms of change (Toth et al., 2016).

This study built on earlier work of two scientifically-validated interventions, namely FMF and PATHS. Similar to several prior FASD interventions (Nash et al., 2015; O'Connor et al., 2006; O'Connor et al., 2016), results from this study document that existing interventions can be effective for children with FASD with appropriate adaptations. Adapting existing interventions improves study efficiency and increases the potential for future dissemination. In the current study, the pre-K/K PATHS curriculum (Domitrovich et al., 2005) was adapted for a small-group setting and curriculum activities were extended to provide varied opportunities for children to practice skills with peers in group. Caregivers were also taught PATHS skills to aid in generalization outside of the group setting.

Study findings suggest that the Families on Track program had the largest impact on the child-related outcomes of emotion regulation and self-esteem, which are two of the major intervention targets of the PATHS program. While large intervention effects were found for these outcomes, children with FASD continued to display impairments relative to normative 
data, consistent with several other FASD treatment studies targeting self-regulation (Nash et al., 2015; Wells et al., 2012). Of particular concern, this study documented declines in selfesteem over time for both groups, with greater declines among children in the comparison group. This is not surprising, given that previous studies of children with FASD as young as 6 years old find these children perceive themselves as "different" from their peers (Stade et al., 2011). Possibly the Families on Track program may have somewhat buffered this decline among children receiving the intervention. While improving children's positive developmental trajectory in any measurable way is a significant (and vital) aim, given the many secondary conditions revealed in natural history data (Streissguth et al., 2004), future intervention efforts should explore whether more intensive or sustained intervention could produce greater benefits in these critical domains.

\section{Limitations}

Study results should be interpreted in context of some limitations. Sample size, although appropriate for study aims of piloting a complex intervention and estimating effect sizes, meant limited statistical power to detect effects. Participants may not be fully representative of families raising children with FASD or PAE. While few exclusion criteria were used, participating families had to engage in an intensive intervention and expect to remain as caregivers throughout the study period. Although all families initially agreed (and despite strong interest), three families declined intervention once selected because of logistical concerns (driving time and cost, scheduling constraints). One child also unexpectedly changed placements, a real-world concern for this clinical population. Notably, no birth parents provided data as primary caregivers in this study (unlike in other FMF trials), though some children had contact, or lived, with a biological parent. Children in this sample were also disproportionately male. Although a wide range of incomes were reported, study families had somewhat higher incomes, on average, than median incomes typical for the area. Some demographic differences between groups, although not statistically significant, could be contributing to outcomes. Future larger-scale trials are needed to replicate findings with more representative samples. It is also possible that greater improvements in the Families on Track group may reflect more intensive contact with this group relative to the comparison group.

There are also limitations for real-world implementation of the Families on Track Program as currently studied. Specialists required over 50 hours of training to be able to deliver the intervention components, which would be a significant cost and time barrier for many clinicians or agencies. In addition, while attendance to child skills groups averaged over $75 \%$ in the current study, sustaining a 30 -week skills group with a single-entry point would be challenging in many community settings (with the exception of schools). The length of sessions (90 minutes) may also not be fully reimbursable. Adaptations to the Families on Track Program to better accommodate these limitations prior to larger-scale study will facilitate future dissemination in real-world settings.

\section{Conclusions and Future Directions}

The Families on Track Integrated Preventive Intervention shows promise for improving child and caregiver outcomes. Findings document additional evidence for the efficacy of the FMF 
Program, and suggest possible benefits for the integration of a child skill building component. Of critical importance to future implementation and dissemination efforts, families reported a high level of program satisfaction, and felt it was effective in meeting their needs. A logical next step is to complete a larger-scale trial with a larger and fully representative sample. Measuring implementation parameters could efficiently inform dissemination and translation to real-world settings (Olson, 2016). Given current limitations in workforce capacity to provide FASD-informed care, exploring translation of these intervention methods into technology-assisted approaches to treatment (e.g., telemedicine, Mobile Health "mHealth") may increase reach to the many families in need of services and decrease challenging barriers to care.

\section{Acknowledgments}

This study was funded by a grant (K01AA020486) from the National Institute on Alcohol Abuse and Alcoholism. The content is solely the responsibility of the authors and does not necessarily represent the official views of the National Institute on Alcohol Abuse and Alcoholism or the National Institutes of Health. We gratefully acknowledge the team of research assistants who participated in data collection, transcription, and coding interviews for this project. Dr. Petrenko also expresses sincere gratitude to the mentors and consultants on her Career Development Award, including Sheree Toth, Heather Taussig, Heather Carmichael Olson, Mary O'Connor, Claire Coles, and Julie Kable, who were generous with their time and advice during the development and implementation of the Families on Track Program. Special thanks also goes to Heather Carmichael Olson for her consultation and feedback on an earlier draft of this manuscript. Most importantly, we thank the courageous and dedicated caregivers, and their children, who participated in this study and without whom these research advances would not be possible.

\section{References}

Abidin, RR. Parenting Stress Index, Fourth Edition: Professional Manual. Psychological Assessment Resources; Lutz, FL: 2012.

Baskin J, Delja JR, Mogil C, Gorospe CM, Paley B. Fetal alcohol spectrum disorders and challenges faced by caregivers: Clinicans' perspectives. J Popul Ther Clin Pharmaco. 2016; 23:e114-e130.

Bertrand J. Interventions for Children with Fetal Alcohol Spectrum Disorders Research Consortium (2009) Interventions for children with fetal alcohol spectrum disorders (FASDs): Overview of findings for five innovative research projects. Res Dev Disabil. 30:986-1006. [PubMed: 19327965]

Carr, A., O'Reilly, M. Service needs of carers for people with intellectual disabilities: Profiles of highneed and low-need groups, in Clinical Psychology in Ireland. In: Carr, A., editor. Empirical Studies of Problems and Treatment Processes in Children and Adolescents. Vol. 3. Edwin Mellen Press; New York: 2000. p. 61-82.

Carr EG, Dunlap G, Horner RH, Koegel RL, Turnbull AP, Sailor W, Anderson JL, Albin RW, Koegel LK, Fox L. Positive behavior support evolution of an applied science. J Posit Behav Interv. 2002; 4:4-16.

Cicchetti D, Toth SL. The past achievements and future promises of developmental psychopathology: the coming of age of a discipline. J Child Psychol \& Psychiat. 2009; 50:16-25. [PubMed: 19175810]

Cohen, J. Statistical power analysis for the behavioral sciences. 2nd. Lawrence Erlbaum Associates; New York: 1988.

Domitrovich CE, Gest SD, Jones D, Gill S, Sanford DeRousie RM. Implementation quality: lessons learned in the context of the Head Start REDI trial. Early Child Res Q. 2010; 25:284-98. [PubMed: 22844183]

Domitrovich, CE., Greenberg, MT., Cortes, R., Kusche, CA. The Preschool PATHS Curriculum. Channing-Bete Publishers; South Deerfield, MA: 2005.

Eyberg, SM., Pincus, D. Eyberg child behavior inventory and sutter-eyberg student behavior inventoryrevised: Professional manual. Psychological Assessment Resources; Lutz, FL: 1999. 
Fabiano GA, Pelham WE Jr, Waschbusch DA, Gnagy EM, Lahey BB, Chronis AM, Burrows-MacLean L. A practical measure of impairment: Psychometric properties of the impairment rating scale in samples of children with attention deficit hyperactivity disorder and two school-based samples. J Clin Child Adolesc Psychol. 2006; 35:369-385. [PubMed: 16836475]

Greenberg, MT., Kusché, C., Mihalic, SF. Promoting Alternative Thinking Strategies (PATHS): Blueprints for Violence Prevention, Book Ten. In: Elliott, DS., editor. Center for the Study and Prevention of Violence. Institute of Behavioral Science, University of Colorado; Boulder, CO: 1998. Blueprints for Violence Prevention Series

Hoyme HE, May PA, Kalberg WO, Kodituwakku P, Gossage JP, Trujillo PM, Buckley DG, Miller JH, Aragon AS, Khaole N, Viljoen DL. A practical clinical approach to diagnosis of fetal alcohol spectrum disorders: clarification of the 1996 institute of medicine criteria. Pediatrics. 2005; 115:39-47. [PubMed: 15629980]

Hoyme HE, Kalberg WO, Elliott AJ, Blankenship J, Buckley D, Marais A, Nursing BC, Manning MA, Robinson LK, Adam MP, Abdul-Rahman O, Jewett T, Coles CD, Chambers C, Jones KL, Adnams CM, Shah PE, Riley EP, Charness ME, Warren KR, May PA. Updated clinical guidelines for diagnosing fetal alcohol spectrum disorders. Pediatrics. 2016; 138

Johnston C, Mash EJ. A measure of parenting satisfaction and efficacy. J Cln Child Psychol. 1989; 18:167-175.

Jones DE, Greenberg M, Crowley M. Early social-emotional functioning and public health: The relationship between kindergarten social competence and future wellness. Am J Public Health. 2015; 105:2283-2290. [PubMed: 26180975]

Kable JA, O'Connor MJ, Olson HC, Paley B, Mattson SN, Anderson SM, Riley EP. Neurobehavioral disorder associated with prenatal alcohol exposure (ND-PAE): Proposed DSM-5 diagnosis. Child Psychiatry Hum Dev. 2015; 47:335-346.

Kam CM, Greenberg MT, Walls CT. Examining the role of implementation quality in school-based prevention using the PATHS curriculum. Prev Sci. 2003; 4:55-63. [PubMed: 12611419]

Kazdin AE, Esveldt-Dawson K, French NH, Unis AS. Problem-solving skills training and relationship therapy in the treatment of antisocial child behavior. J Consult Clin Psychol. 1987; 55:76-85. [PubMed: 3571662]

Kodituwakku PW. Neurocognitive profile in children with fetal alcohol spectrum disorders. Dev Dis Res Rev. 2009; 15:218-224.

Koegel, LKE., Koegel, RL., Dunlap, GE. Positive behavioral support: Including people with difficult behavior in the community. Paul H Brookes Publishing; Baltimore, MD: 1996.

Kreutzer, JS., Camplair, P., Waaland, P. Family Needs Questionnaire. Medical College of Virginia, Rehabilitation Research and Training Center on Severe Traumatic Brain Injury; Richmond, VA: 1988.

Kully-Martens K, Denys K, Treit S, Tamana S, Rasmussen C. A review of social skills deficits in individuals with fetal alcohol spectrum disorders and prenatal alcohol exposure: profiles, mechanisms, and interventions. Alcohol Clin Exp Res. 2012; 36:568-76. [PubMed: 22017360]

Kusche, CA., Greenberg, MT. The PATHS (Promoting Alternative Thinking Strategies) Curriculum. Channing-Bete Co; South Deerfield, MA: 1994.

Larsen DL, Attkisson CC, Hargreaves WA, Nguyen TD. Assessment of client/patient satisfaction: Development of a general scale. Eval Program Plann. 1979; 2:197-207. [PubMed: 10245370]

Mattson SN, Crocker N, Nguyen TT. Fetal alcohol spectrum disorders: neuropsychological and behavioral features. Neuropsychol Rev. 2011; 21:81-101. [PubMed: 21503685]

May PA, Baete A, Russo J, Elliott AJ, Blankenship J, Kalberg WO, Buckley D, Brooks M, Hasken J, Abdul-Rahman O, Adam MP, Robinson LK, Manning M, Hoyme HE. Prevalence and characteristics of fetal alcohol spectrum disorders. Pediatrics. 2014; 134:855-866. [PubMed: 25349310]

Measelle JR, Ablow JC, Cowan PA, Cowan CP. Assessing young children's views of their academic, social, and emotional lives: An evaluation of the self-perception scales of the Berkeley Puppet Interview. Child Dev. 1998; 69:1556-1576. [PubMed: 9914640] 
Moffitt TE, Arseneault L, Belsky D, Dickson N, Hancox RJ, Harrington H, Caspi A. A gradient of childhood self-control predicts health, wealth, and public safety. Proc Natl Acad Sci. 2011; 108:2693-2698. [PubMed: 21262822]

Morris SB. Estimating effect sizes from pretest-posttest-control group designs. Organ Res Meth. 2008; 11:364-86.

Nash K, Stevens S, Greenbaum R, Weiner J, Koren G, Rovet J. Improving executive functioning in children with fetal alcohol spectrum disorders. Child Neuropsychol. 2015; 21:191-209. [PubMed: 25010354]

O'Connor MJ, Frankel F, Paley B, Schonfield AM, Carpenter E, Laugeson EA, Marquardt R. A controlled social skills training for children with fetal alcohol spectrum disorders. J Consult Clin Psychol. 2006; 74:639-48. [PubMed: 16881771]

O'Connor MJ, Quattlebaum J, Casteneda M, Dipple KM. Alcohol intervention for adolescents with fetal alcohol spectrum disorders: Project Setp Up, a treatment development study. Alcohol Clin Exp Res. 2016; 40:1744-1751. [PubMed: 27219498]

Olson, HC., Montague, RA. An innovative look at early intervention for children affected by prenatal alcohol exposure. In: Adubato, S., Cohen, D., editors. Prenatal Alcohol Use and FASD: A Model for Diagnosis, Assessment and New Directions in Research and Multimodal Treatment. Bentham Science Publishers; Potomac, MD: 2011. p. 64-107.

Olson HC, Oti R, Gelo J, Beck S. "Family matters:” Fetal alcohol spectrum disorders and the family. Dev Disabil Res Rev. 2009; 15:235-249. [PubMed: 19731388]

Olson HC. A renewed call to action: the need for systematic research on interventions for FASD. Alcohol Clin Exp Res. 2016; 40:1817-21. [PubMed: 27453398]

Petrenko CL. Positive behavioral interventions and family support for fetal alcohol spectrum disorders. Curr Dev Disord Rep. 2015; 2:199-209. [PubMed: 26380802]

Petrenko CLM, Tahir N, Mahoney EC, Chin NP. A qualitative assessment of program characteristics for preventing secondary conditions in individuals with fetal alcohol spectrum disorders. J Popul Ther Clin Pharmacol. 2014a; 21:e246-e259. [PubMed: 25032710]

Petrenko CL, Tahir N, Mahoney EC, Chin NP. Prevention of secondary conditions in fetal alcohol spectrum disorders: Identification of systems-level barriers. Matern Child Health J. 2014b; 18:1496-1505. [PubMed: 24178158]

Petrenko CLM, Pandolfino ME, Roddenbery R. The association between parental attributions of misbehavior and parenting practices in caregivers raising children with prenatal alcohol exposure: A mixed-methods study. Res Dev Disabil. 2016; 59:255-267. [PubMed: 27662038]

Popova, S., Lange, S., Burd, L., Rehm, J. Oxford Handbooks Online. New York: 2016. Burden and social cost of fetal alcohol spectrum disorders.

Reid N, Dawe S, Shelton D, Harnett P, Warner J, Armstrong E, LeGros K, O'Callaghan F. Systematic review of fetal alcohol spectrum disorder interventions across the lifespan. Alcohol Clin Exp Res. 2015; 39:2283-95. [PubMed: 26578111]

Ryan DM, Bonnett DM, Gass CB. Sobering thoughts: Town hall meetings on fetal alcohol spectrum disorders. Am J Public Health. 2006; 96:2098-2101. [PubMed: 17077397]

SAMHSA-HRSA Center for Integrated Health Solutions. A standard framework for levels of integrated health care. SAMHSA-HRSA Center for Integrated Health Solutions; Washington D.C: 2013.

Sampson PD, Streissguth AP, Bookstein FL, Little RE, Clarren SK, Dehaene P, Hanson JW, Graham JM. Incidence of fetal alcohol syndrome and prevalence of alcohol-related neurodevelopmental disorder. Teratology. 1997; 56:317-326. [PubMed: 9451756]

Shields A, Cicchetti D. Emotion regulation among school-age children: The development and validation of a new criterion Q-sort scale. Dev Psychol. 1997; 33:906-916. [PubMed: 9383613]

Sroufe LA. The promise of developmental psychopathology: past and present. Dev Psychopathol. 2013; 25:1215-1224. [PubMed: 24342836]

Stade B, Beyene J, Buller K, Ross S, Patterson K, Stevens B, Sgro M, Ungar W, Watson W, Koren G. Feeling different: the experience living with fetal alcohol spectrum disorder. J Popul Ther Clin Pharmacol. 2011; 18:475-485. 
Streissguth AP, Bookstein FL, Barr HM, Sampson PD, O'Malley K, Young JK. Risk factors for adverse life outcomes in fetal alcohol syndrome and fetal alcohol effects. J Dev Behav Pediatr. 2004; 25:228-238. [PubMed: 15308923]

Toth, SL., Petrenko, CLM., Gravener Davis, J., Handley, ED. Advances in Prevention Science: A Developmental Psychopathology Perspective. In: Cicchetti, D., editor. Developmental Psychopathology. Vol. 4. Wiley; New York: 2016.

Wells AM, Chasnoff IJ, Schmidt CA, Telford E, Schwartz LD. Neurocognitive habilitation therapy for children with fetal alcohol spectrum disorders: An adaptation of the alert program ${ }^{\circledR}$. Am J Occup Ther. 2012; 66:24-34. [PubMed: 22251828] 


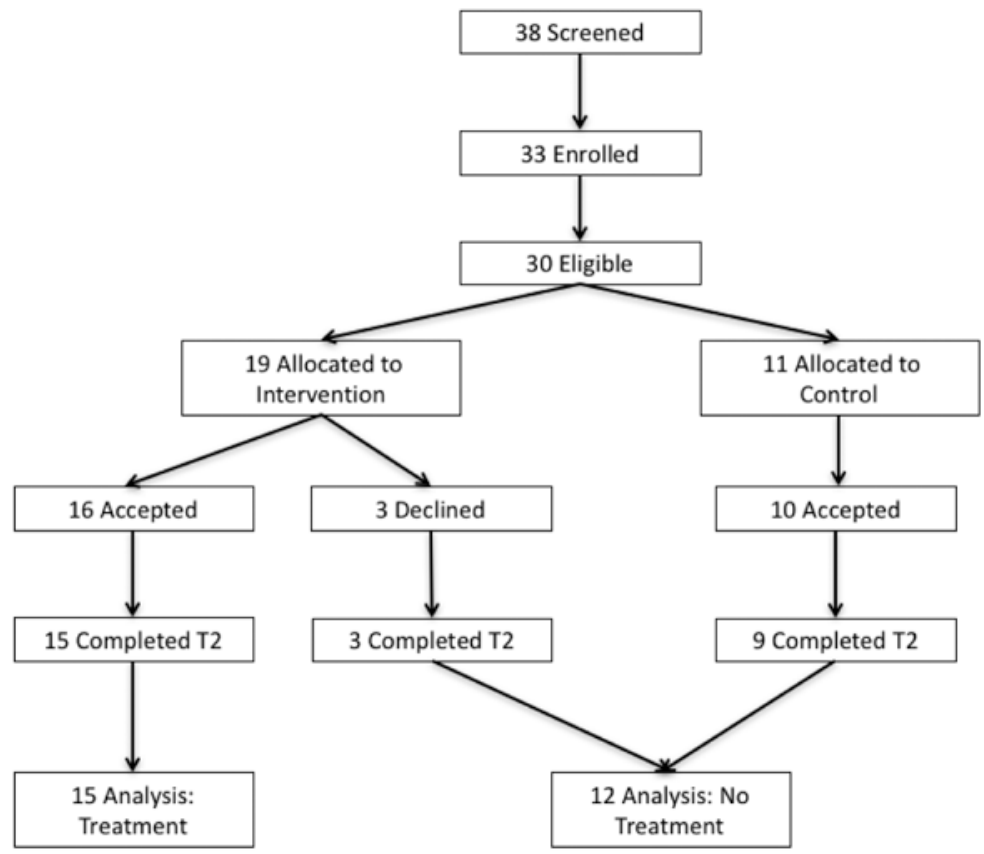

Figure 1.

Study flow diagram. 


\section{Table 1}

Battery used in neuropsychological assessments for all study participants.

\begin{tabular}{ll}
\hline Domain & Test \\
\hline General Cognitive Ability & Differential Ability Scales, Second Edition (DAS-II) \\
\hline Academic Achievement & Woodcock-Johnson Tests of Achievement, Third Edition Brief Battery \\
\hline Sensory Motor & $\begin{array}{l}\text { NEPSY-II Visuomotor Precision } \\
\text { Short Sensory Profile }\end{array}$ \\
\hline Language / Social Communication & Children's Communication Checklist, Second Edition \\
\hline \multirow{2}{*}{ Learning and Memory } & DAS-II Recognition of Pictures \\
\hline & DAS-II Recall of Digits Forward \\
& NEPSY-II Narrative Memory \\
\hline Executive Functioning & DAS-II Recall of Digits Backward \\
& NEPSY-II Inhibition \\
\hline Behavior Functioning & NEPSY-II Word Generation \\
\hline Adaptive Functioning & Behavior Rating Inventory of Executive Function \\
\hline
\end{tabular}




\section{Table 2}

Families on Track session order

\begin{tabular}{|c|c|c|}
\hline Week & Parent Sessions & Child Skills Group Sessions \\
\hline 1 & Engagement and Functional Assessment & Unit 1, lessons 1-3 (modified) - PATHS Animals, Rules, Kid for Today \\
\hline 2 & & Unit 1, lesson 4 - Compliments I \\
\hline 3 & $\begin{array}{l}\text { Functional Assessment and Understanding Caregiver } \\
\text { Needs }\end{array}$ & Unit 2, lesson 6- Happy \\
\hline 4 & & Unit 2, lesson $7-S a d$ \\
\hline 5 & Directed Observations & Unit 2, lesson 8 - Twiggle Makes Friends \\
\hline 6 & & Unit 2, lesson 9 - Compliments II \\
\hline 7 & Creating 1st Behavior Support Plan ("Brainstorming") & Unit 3, lesson 10 - Mad or Angry I \\
\hline 8 & & Unit 3, lesson 11 - Scared or Afraid \\
\hline 9 & Feedback & Unit 3, lesson $12-$ My Feelings \\
\hline 10 & & Unit 3, lesson $13-$ Mad II \\
\hline 11 & Understanding the Whole Child / Calming for Kids & Unit 4, lesson 14 - Twiggle Learns to do Turtle, Part I \\
\hline 12 & & Unit 4, lesson 15 - Twiggle Learns to do Turtle, Part II \\
\hline 13 & Reframing & Unit 4, Lesson 16 - Turtle Technique Review \\
\hline 14 & & Unit 4, Lesson 17 - Appropriate Turtles I \\
\hline 15 & Accommodations & Unit 4, Lesson 18 - Appropriate Turtles II \\
\hline 16 & & Unit 4, Lesson 19 - Calm or Relaxed \\
\hline 17 & Creating $2^{\text {nd }}$ Behavior Support Plan & Unit 5, Lesson 20 - Sharing and Caring $I$ \\
\hline 18 & & Unit 5, Lesson 21 - Sharing and Caring II \\
\hline 19 & Self Care ( \& a Pizza) & Unit 5, Lessons 22 \& 23 - Twiggle's Special Day, Advanced Compliments \\
\hline 20 & & Unit 6, Lesson 25 - Making Choices \\
\hline 21 & Individualized Session & Unit 6, Lesson 26 - Solving Problems \\
\hline 22 & & Unit 6, Lesson 27 - Solving Problems with Friends \\
\hline 23 & Individualized Session & Unit 7, Lesson 28 - Comfortable and Uncomfortable \\
\hline 24 & & Unit 7, Lesson 29 - Different Types of Feelings \\
\hline 25 & Looking Forward & Unit 7, Lesson $30-$ Excited \\
\hline 26 & & Unit 7, Lesson 32 - Frustrated \\
\hline 27 & Advocacy \& Linkages & Unit 7, Lesson 33 - Proud \\
\hline 28 & & Unit 8, Lesson $34-$ Love \\
\hline 29 & Support \& Integration & Unit 9, Session 43 - Ending and Transitioning \& Review \\
\hline 30 & & Party \\
\hline
\end{tabular}




\section{Table 3}

\section{Participant demographics}

\begin{tabular}{|c|c|c|}
\hline Variable & Intervention $(n=16)$ & Comparison $(n=14)^{*}$ \\
\hline Child age, mean (SD) & $6.52(1.31)$ & $6.59(1.28)$ \\
\hline Child sex, $\%$ male $[\mathrm{n}]$ & $62.5[10]$ & $92.9[13]$ \\
\hline \multicolumn{3}{|l|}{ Child race/ethnicity $\%[\mathrm{n}]^{\wedge}$} \\
\hline Hispanic/Latino & $12.5[2]$ & $14.3[2]$ \\
\hline African American/Black & $25.0[4]$ & $21.4[3]$ \\
\hline Native American & $6.3[1]$ & $0.0[0]$ \\
\hline Asian & $6.3[1]$ & $0.0[0]$ \\
\hline Caucasian/White & $81.3[13]$ & $85.7[12]$ \\
\hline FAS/pFAS diagnosis, $\%$ [n] & $50.0[8]$ & $35.7[5]$ \\
\hline Cognitive ability, mean (SD) & $90.25(11.52)$ & $88.93(14.86)$ \\
\hline Adaptive functioning, mean (SD) & $77.25(19.39)$ & $72.21(17.22)$ \\
\hline \multicolumn{3}{|l|}{ Placement type $\%$ [n] } \\
\hline Foster/adoptive & $81.3[13]$ & $71.4[10]$ \\
\hline Relative & $18.8[3]$ & $28.6[4]$ \\
\hline Caregiver age, mean $[\mathrm{SD}]$ & $45.77(8.97)$ & $48.19(8.19)$ \\
\hline Caregiver sex, $\%$ female $[\mathrm{n}]$ & $93.8[15]$ & $78.6[11]$ \\
\hline \multicolumn{3}{|l|}{ Caregiver race/ethnicity $\%[\mathrm{n}]^{\wedge}$} \\
\hline Hispanic/Latino & $6.3[1]$ & $0.0[0]$ \\
\hline African American/Black & $6.3[1]$ & $0.0[0]$ \\
\hline Native American & $6.3[1]$ & $7.1[1]$ \\
\hline Caucasian/White & $87.5[14]$ & $100[14]$ \\
\hline \multicolumn{3}{|l|}{ Caregiver marital status \% [n] } \\
\hline Married/Living with partner & $68.8[11]$ & $78.5[11]$ \\
\hline Single parent (divorced, widowed, never married) & $31.2[5]$ & $21.5[3]$ \\
\hline \multicolumn{3}{|l|}{ Caregiver educational attainment $\%[\mathrm{n}]$} \\
\hline High school degree / GED & $6.3[1]$ & $35.7[5]$ \\
\hline Associates degree & $18.8[3]$ & $35.7[5]$ \\
\hline Bachelor's degree & $31.3[5]$ & $21.4[3]$ \\
\hline Graduate/Professional degree & $37.6[6]$ & $7.1[1]$ \\
\hline Family income, mean (SD) & $\$ 90,312(49,378)$ & $\$ 74,128(28,386)$ \\
\hline
\end{tabular}

Alcohol Clin Exp Res. Author manuscript; available in PMC 2018 July 01. 


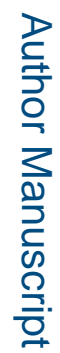

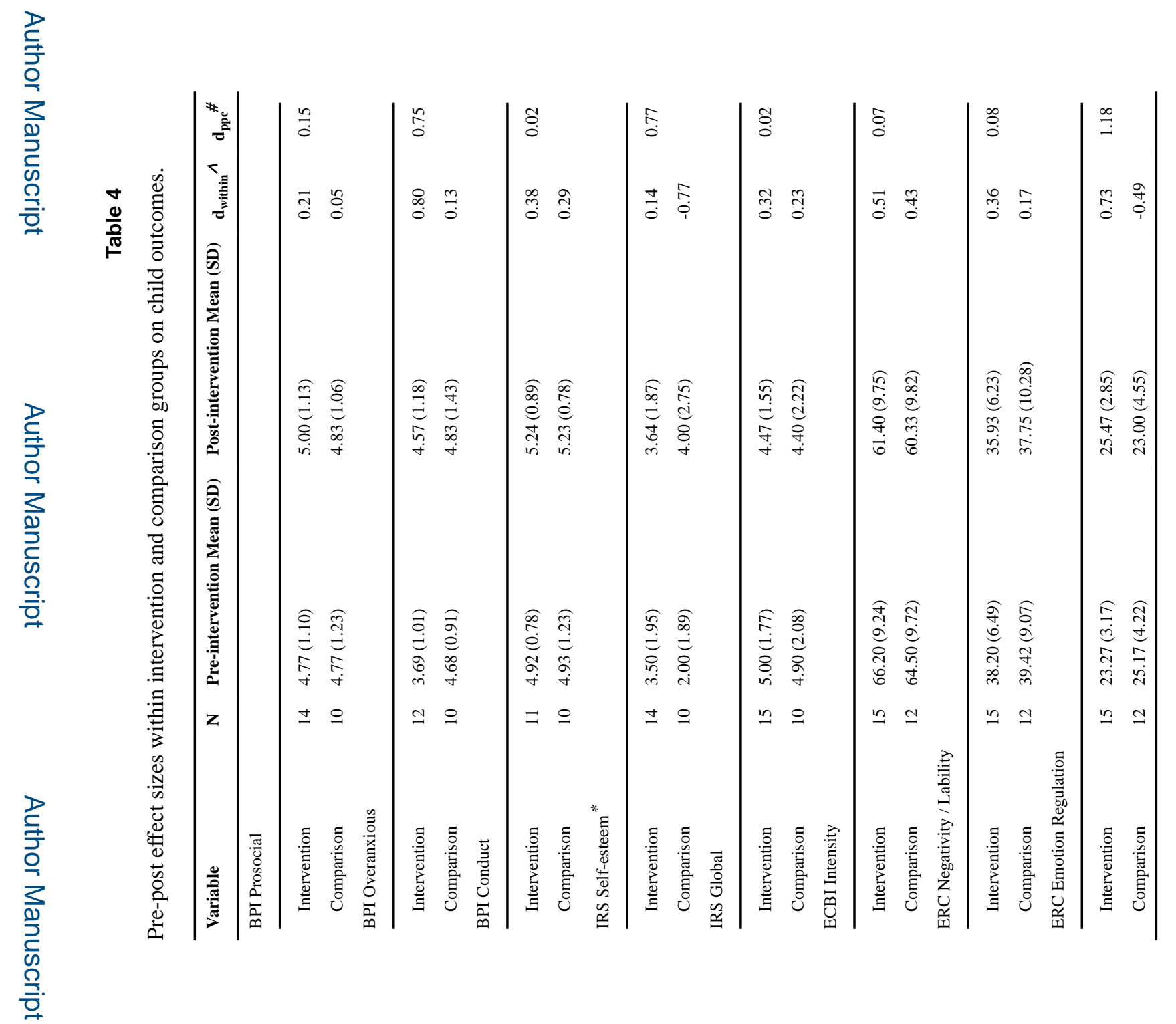


Petrenko et al.

Page 24

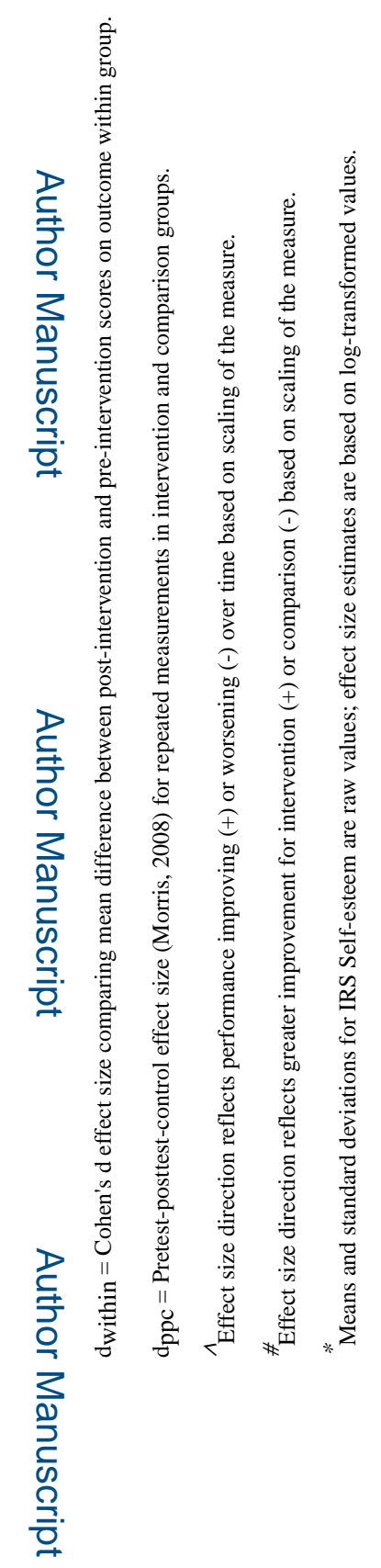

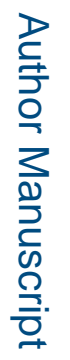

Alcohol Clin Exp Res. Author manuscript; available in PMC 2018 July 01. 


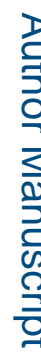

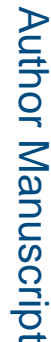

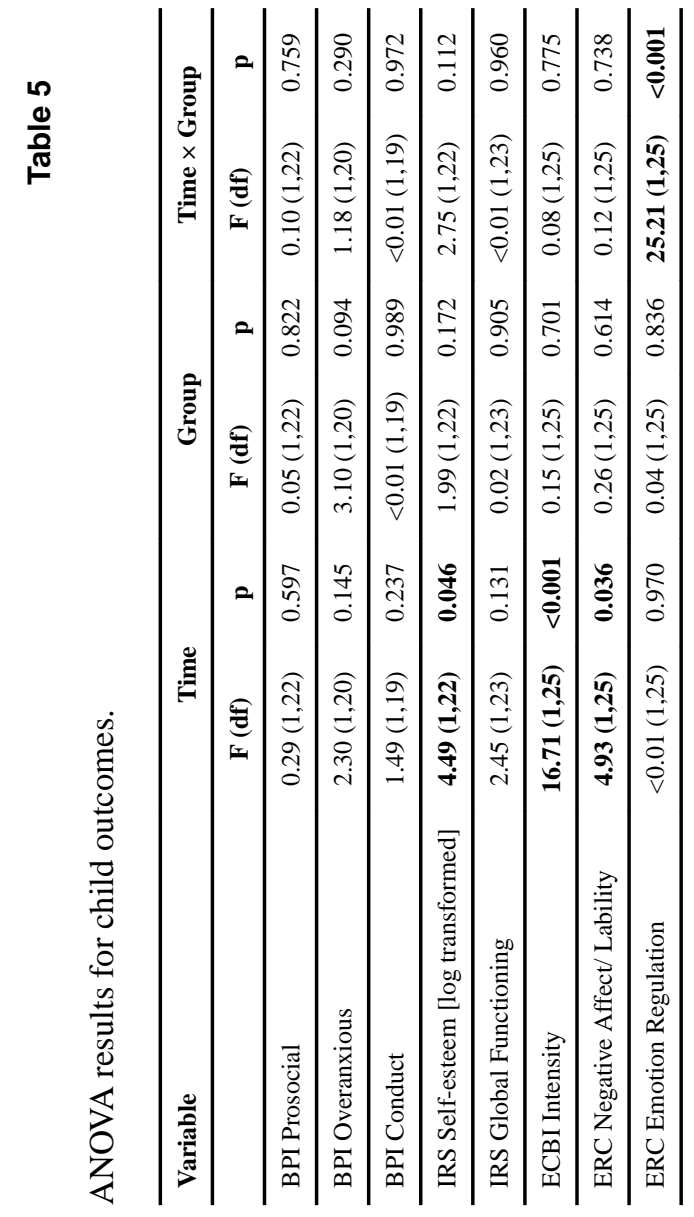




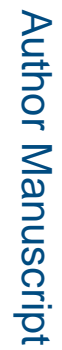

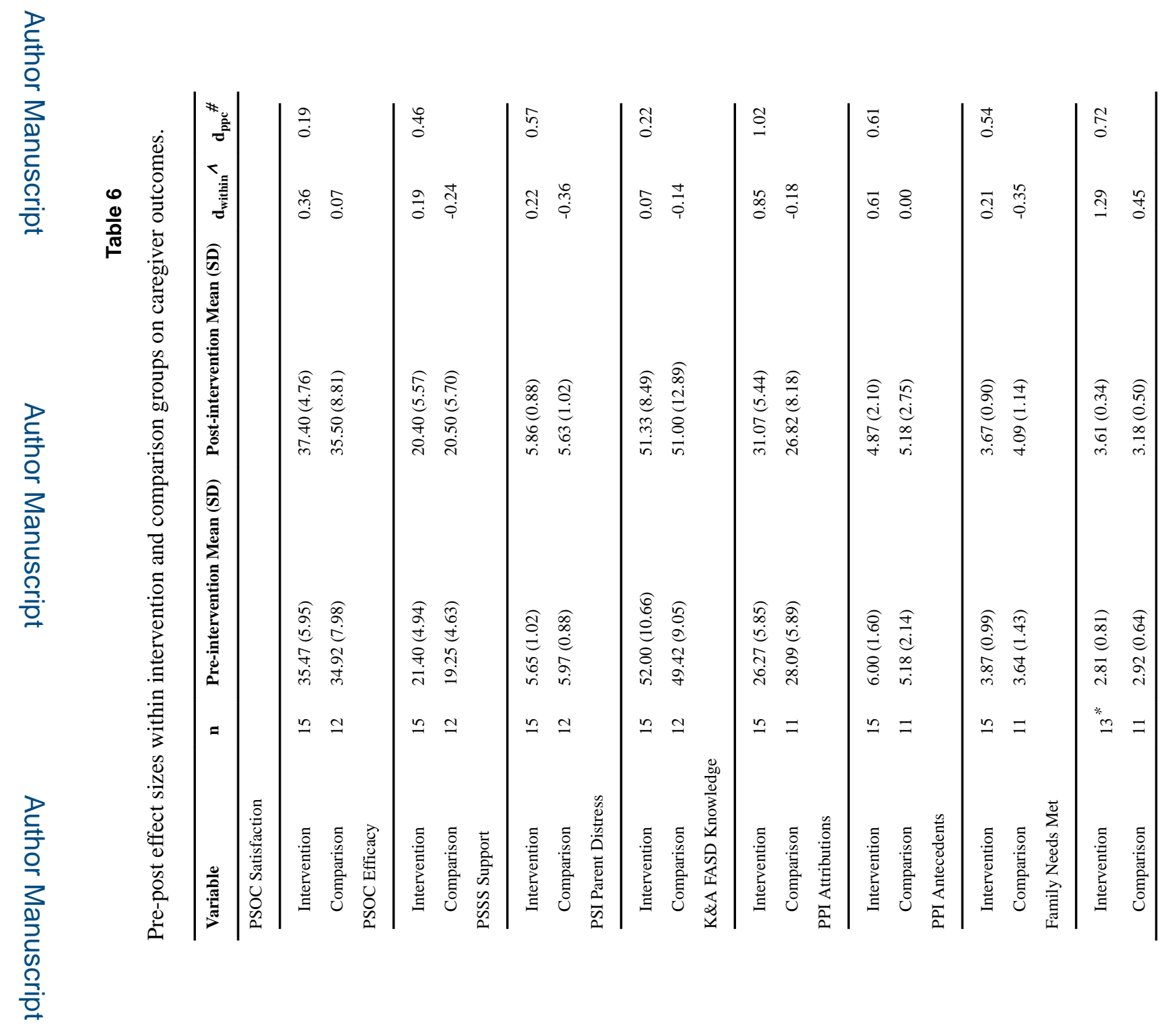


Petrenko et al.

Page 27

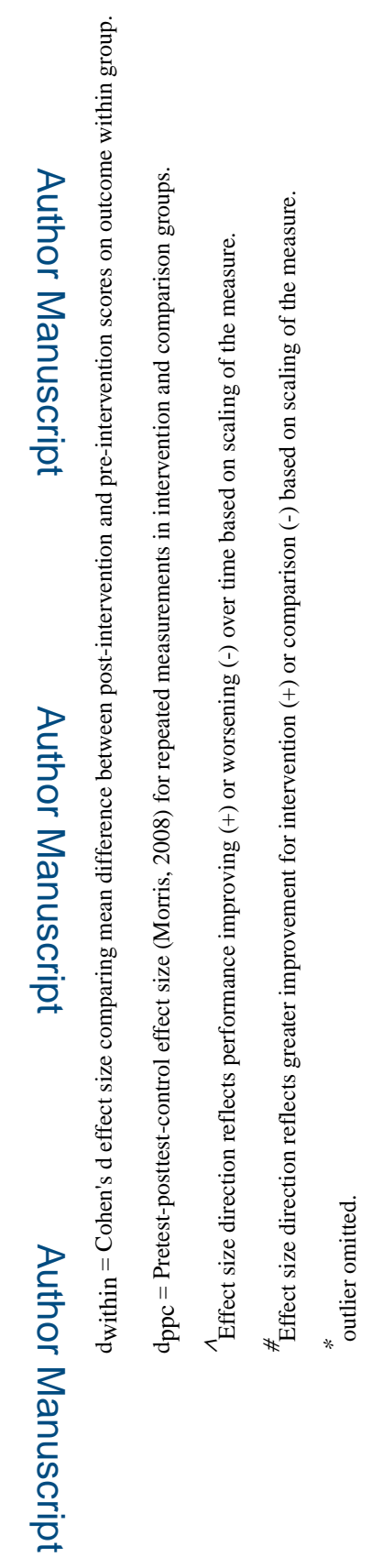

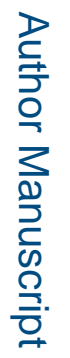

Alcohol Clin Exp Res. Author manuscript; available in PMC 2018 July 01. 


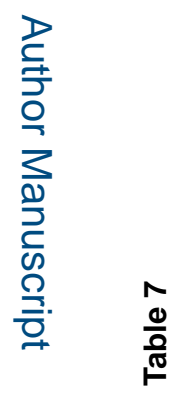

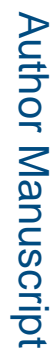

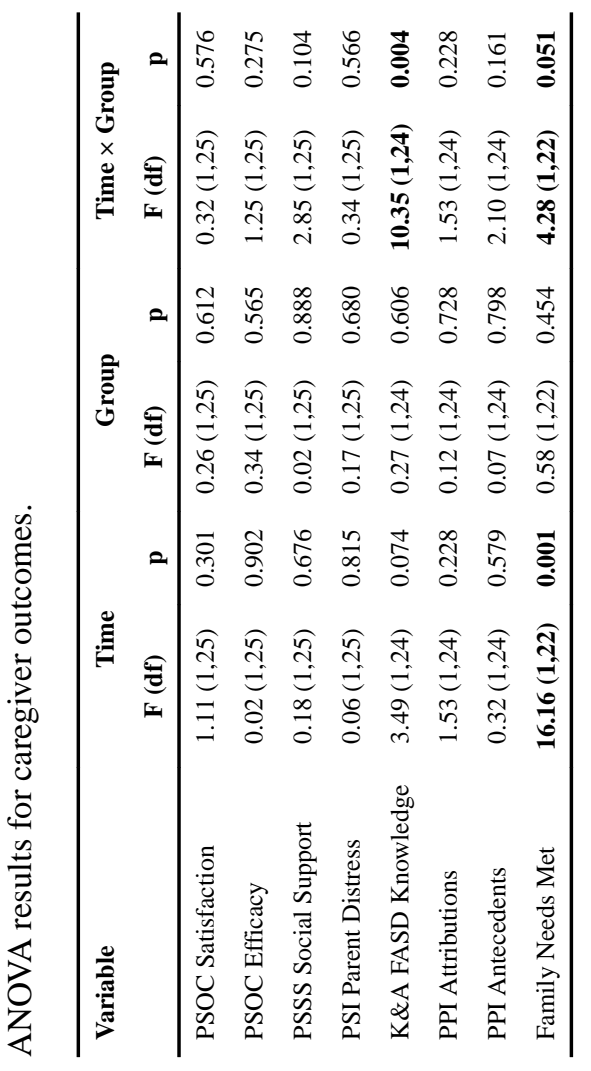

Alcohol Clin Exp Res. Author manuscript; available in PMC 2018 July 01. 